Rev. Elev. Méd. vét. Pays trop., 1966, 19, 3 (275-276).

\title{
Note préliminaire sur la présence de Fasciola gigantica à Madagascar
}

\author{
par P. DAYNES
}

La fasciolose des ruminants, infestation grave due à la présence de trématodes du genre Fosciola dans les canaux biliaires, est très répandue dans le monde. Dans les pays tempérés c'est Fasciola hepatica, la grande douve, qui est en cause alors que dans les pays chauds on trouve généralement Fosciola gigantica, la douve géante.

Jusqu'à ces derniers temps, Madagascar étaif considérée par tous comme exempte de fasciolose. On avait bien rencontré Fosciola hepatica chez des ovins importés d'Europe, en particulier en 1929 et 1930 (et peut-être dès 1904), mais ces parasites ne s'étaient pas multipliés dans l'lle (POISSON H., 1929 Bull. Path. exo p. 521).

Récemment, fin avril 1966, une vache appartenant à B., au Centre de K., canton de Mahasolo, district de Tsiroanomandidy, province de Tananarive, morte subitement, présentait selon te rapport d'autopsie de nombreux «parasites dans les canaux hépatiques, d'où dégénérescence totale du foie ». D'après l'agent ayant pratiqué l'autopsie. I'animal était maigre et anémié. Le foie de couleur pâle présentait des taches blanchâtres en surface. mais pas de trace d'angiocholite visible de l'extérieur de l'organe. Ces parasites des canaux hépatıques ont été reconnus comme étant Fasciola gigantico que I'on n'avait encore jamais signalé à Madagascar. L'animal en cause, était une vache de race Rana, race laitière des environs de Tananarive, achetée 2 ans plus tôt à un éleveur de la région péri-tananarıvienne. Depuis 2 ans cet animal fréquentait un pâturage composé de pentes et plateaux ferralitique, à Heteropogon. Hyparrhenia et Aristida ainsi qu'un bas fond tourbeux à Cyperacées dominantes avec Leersia hexandra et Panicum glanduliferum. Le $\mathrm{pH}$ du bas fond est acide ( $\mathrm{pH}$ de l'ordre de 4.5).
Au préalable (c'est-à-dire pendanł 3 ans environ, cet animal étant âgé de 5 ans) la vache vivait dans une région identique, au nord de Tananarive, chez D. à Mahiłsy.

Le troupeau auquel appartenait cette vache, composé de 101 vaches ou génisses et 2 taureaux a immédiatement fait l'objet d'un examen coproscopique.

Au total 8 animaux ont présenté dans leurs excréments des cufs pouvant faire soupçonner la fasciolose. Ces cufs avaient des tailles variant de 160 à 180 microns de long sur 80 à 90 microns de large. Leur couleur jaunâtre ajoutée à leurs dimensions les différencient des cuffs des trématodes généralement rencontrés (Paramphistomum, Cotylophoron ef Carmyerius).

F. gigantica a peut-être été importée avec des bovins provenant d'Amérique ou d'Afrique (Importations de bétal brahman du Texas et de bétail Sahiwal du Kenya, ces bétails ayant été précisément introduits dans le Centre de K.).

Le fait que $F$. gigantica aif été rencontrée sur un anımal né à Madagascar prouve que le cycle du parasite s'est fermé sur place. Des recherches sur la situation actuelle de la fasciolose des bovins et sur le vecteur possible (Lymnaea hovarum ?) sont entreprises.

Notons la présence en relativement grand nombre de mollusques dans le petit ruisseau de réunion des eaux du bas-fond intéressé. Nous trouvons de nombreux Bionphalario et de presqu'aussi nombreux Lymnaea hovarum infestés de formes larvaires de trématodes divers.

\section{Institut d'Elevage ef de Médecine Vétérinaire des Pays Tropicaux.}

Laboratoire central de l'Elevage de Tonanarive. 


\section{SUMMARY}

Preliminary note on the presence of Fasciola gigantica in Madagascar

The presence of Fasciola gigantica in cattle born in Madagascar is reported for the first time by the author.

\section{RESUMEN}

Nota preliminar sobre la presencia de Fosciola gigantica en Madagascar

Por la primera vez se nota en Madagascar la presencia de Fasciola gigantica en un bovino nacido en el pois. 